\title{
Managing surgical conflicts of interest
}

W

hen "conflict of interest" and "medicine" appear in the same sentence, what follows is likely about financial ties between physicians and drug companies. But there is another commonplace relationship in medicine that, though explored less often, is nonetheless rife with potential conflicts of interest: the one between surgeons and medical-device makers.

Two recently settled court cases in California highlight the problems that can arise when surgeons are paid hundreds of thousands of dollars by companies that manufacturer medical devices used in surgery. Early this summer, as reported in the Los Angeles Times, the University of California, Los Angeles (UCLA) paid $\$ 8.5$ million (US) to settle two lawsuits against a spine surgeon who worked for the university.

The plaintiffs, who suffered postsurgery complications including pain and the need for more operations, alleged that Dr. Jeffrey Wang used a bonegrowth product made by Medtronic for an off-label use that wasn't approved by the US Food and Drug Administration (FDA). They also claimed Wang failed to disclose that Medtronic had paid him more than $\$ 275000$ for product royalties, lecturing and consulting. UCLA, Wang and Medtronic all denied wrongdoing, the Los Angeles Times reported.

'Dr. Wang violated my clients' trust by concealing his financial and research relationships with the manufacturers and devices he implanted in my clients," Robert Vaage, the San Diego lawyer who represented both plaintiffs, said in an email to CMAJ. "Dr. Wang's use of the devices resulted in severe and permanent injuries to both of my clients. ... Given that their health cannot be restored, they are satisfied with the outcome of these cases, and hope that these results will prevent others from being injured as they were."

Vaage also said that UCLA had been on notice for at least two years before the surgeries (performed in 2009 and 2010) that Wang and other

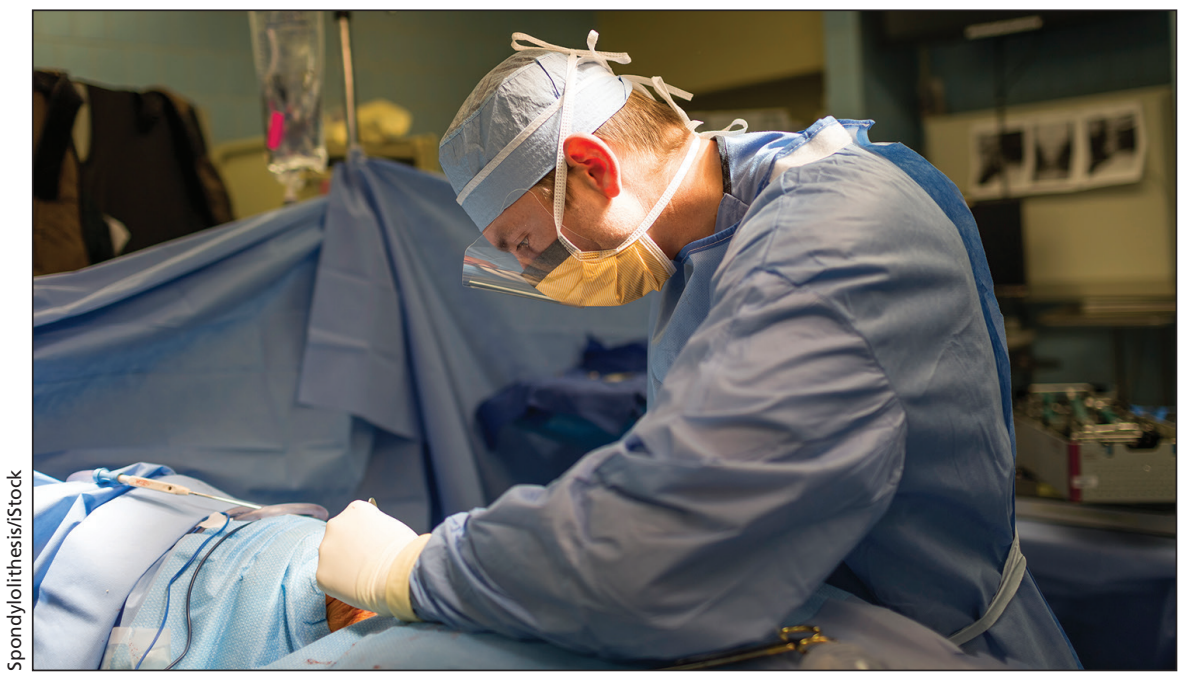

Are surgeons with financial ties to medical-device makers biased toward using those companies' products?

surgeons were violating university policies requiring disclosure of financial relationships with device makers, yet did nothing to enforce the policies.

In response to Vaage's comments, UCLA Health forwarded CMAJ a prepared statement on the lawsuits. The school settled the lawsuits "so that UCLA Health and the David Geffen School of Medicine at UCLA could move forward with their ongoing commitment to excellence in patient care, research, education and community service," said the statement, sent to CMAJ by Phil Hampton, director of media relations for UCLA Health and the medical school. "UCLA Health takes seriously its duty to manage relationships with industry in a responsible manner, and we will continue to make our high standards clear and provide appropriate guidance to faculty and staff."

These lawsuits raise a number of ethical concerns about conflicts of interest, patient harm and compromised patient autonomy, according to Jane Johnson, a fellow in the philosophy department at Macquarie University in Sydney, Australia, who has studied the ethics of surgical innovation.

"Establishing a causal link between manufacturer payments and the kind of surgical choices that result in patient harm in any one case is problematic," Johnson wrote in an email to CMAJ. "But research shows conflicts, even involving small gifts, generate unconscious bias and feelings of reciprocity in the beneficiary. And because surgery by its very nature involves uncertainty and subjective judgement [sic], there is significant scope for such bias to slip into and impact decision-making."

In a 2014 paper in BMC Medical Ethics, Johnson explored how surgical conflicts of interested could be better managed. The suggestions include developing institutional policies to prohibit activities that don't contribute to improving patient care and creating procedures to educate people about the nature and effects of conflicts of interest.

"Addressing these issues is by no means straightforward and needs to go well beyond the kind of disclosure often touted as a solution," said Johnson. "A surgical culture which encourages and supports second opinions, third-party consent and the employment of patient advocates may go some way to meeting concerns about bias." - Roger Collier, CMAJ

CMAJ 2016. DOI:10.1503/cmaj.109-5320 